\title{
Identifying Precancerous Changes in a Woman with Dense Breasts and Breast Implants
}

\author{
Richard M Fleming ${ }^{1 *}$, Matthew R Fleming ${ }^{1}$, Tapan K Chaudhuri ${ }^{2}$ and William C Dooley ${ }^{3}$ \\ ${ }^{1}$ FHHI-Omnific Imaging-Camelot, USA \\ ${ }^{2}$ Eastern Virginia Medical School, USA \\ ${ }^{3}$ Oklahoma University Health Science Center, USA
}

\begin{abstract}
Evaluation of women with dense breasts is limited using mammography due to the increased fibro glandular tissue present in 50\% of all women. Measurement of regional blood flow (RBF) and metabolic differences differentiate tissue types and can be used in women with dense breasts to determine if inflammation, precancerous changes or breast cancer is present. It is also now appreciated that women with breast implants have an increased risk of developing inflammatory reactions - both within the breast and systemically - as well as cancer. We present once such case of a woman with breast irregularities, dense breasts and breast implants, evaluated by FMTVDM*.
\end{abstract}

KEYWORDS: FMTVDM; Breast cancer; Mammography; Dense breasts; Breast Implants

ABBREVIATIONS: RBF: Regional Blood Flow; ROI: Regions of Interest; FMTVDM: The Fleming Method for Tissue and Vascular Differentiation and Metabolism

\section{INTRODUCTION}

One in every eight women will be diagnosed with breast cancer at least once in their lifetime. Half (50\%) of all women have what is defined as dense breasts - breasts associated with $50 \%$ or more of their breast being either supportive (fibrous) or glandular tissue. The remaining $50 \%$ of women have breasts with less fibro glandular tissue and are considered to have non-dense breasts; i.e. they have more fatty tissue. Mammographic x-rays more easily penetrate fatty breast tissue - in contrast to fibro glandular tissue.

The risk of inflammatory changes, potential autoimmune diseases and breast cancer in women with breast implants is also now well recognized [1-6]. Given the prevalence of breast implants and the likelihood that breast implants will not become a thing of the past, it is important that clinicians be able to monitor the overall health of the women and men with breast implants by measuring changes in breast health [7-9] on a Health-Spectrum as shown in Figure 1.
We present a case of a woman with both dense breast and breast implants that noticed breast irregularities and requested further measurement of metabolic and regional blood flow (RBF) differences using FMTVDM.

\section{PATIENT STUDY}

Following standardized preparation and introduction to the nuclear imaging laboratory and following the signing of informed consent per laboratory protocol, a middle-aged Caucasian woman presented for evaluation using FMTVDM [7-9]. She had noted several breast irregularities and had been told following prior mammograms that she had dense breasts. Years earlier she had elected for breast implants for augmentation purposes. She had no known genetic concerns, was a non-smoker and had not previously taken hormone therapy for any purpose.
Quick Response Code:

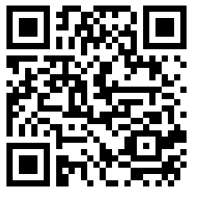

Address for correspondence: Richard M Fleming, FHHI-Omnific Imaging-Camelot, USA

Received: November 22, 2019 Published: November 26, 2019

How to cite this article: Richard MF, Matthew RF, Tapan KC, William CD. Identifying Precancerous Changes in a Woman with Dense Breasts and Breast Implants. 2019 - 1(3) OAJBS.ID.000118. DOI: 10.38125/OAJBS.000118 


\section{Quantification of the Changes in "Health-Spectrum"

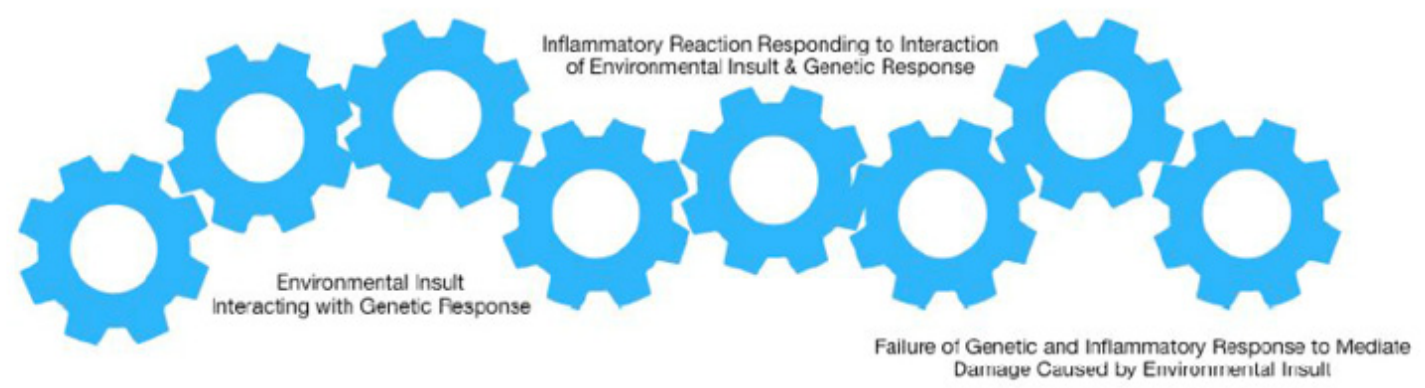

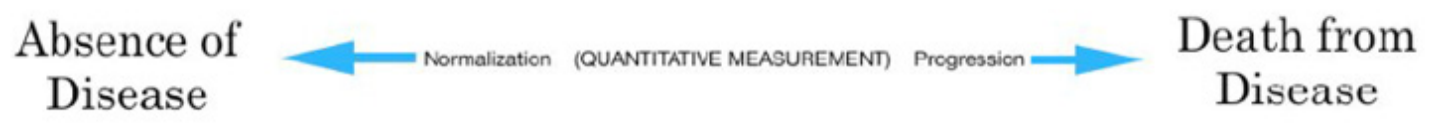

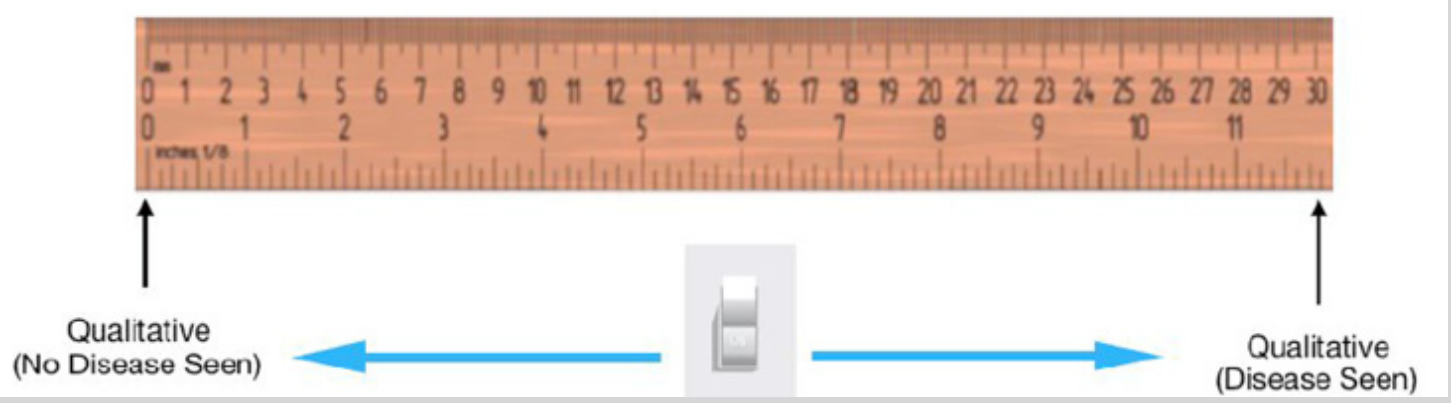

Figure 1: Progression or regression of health across a health-spectrum is a measurable continuum of transitional changes occurring over time resulting from the interaction of the specific person's cellular genetic composition and the environmental influences impacting specific cells and tissue [9].

The result of her FMTVDM is presented in Figure 2, showing several drawn regions of interest (ROIs) with measurements obtained for regional blood flow (RBF) and metabolism. The maximal count for the right breast of 317 is consistent with precancerous tissue [7-9] and matched the region identified by the patient as being irregular.

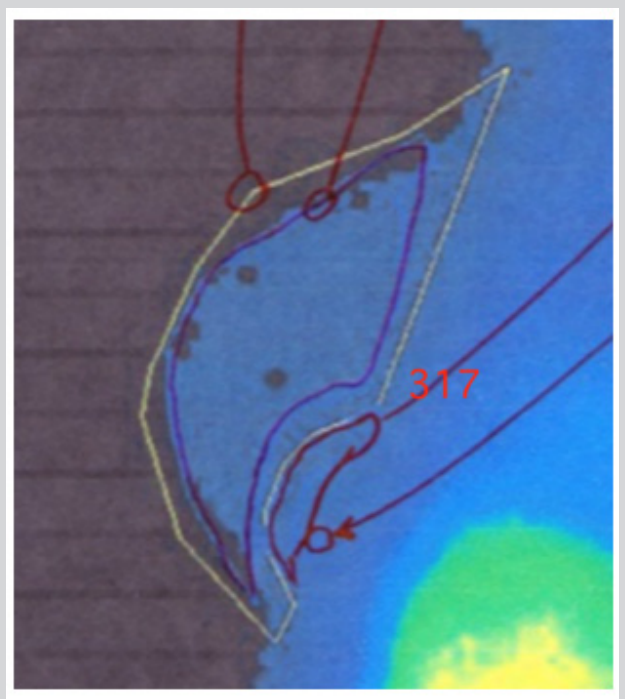

Figure 2: FMTVDM results for a woman with precancerous breast tissue change with breast implants.

\section{DISCUSSION}

Eighty to ninety percent $(80-90 \%)$ of the time, breast irregularities are noted by women and brought to the attention of their physician - as was the case in this instance. The use of mammography is unreliable in women with dense breasts and additionally complicated by the inclusion of breast implants.

FMTVDM measurement of metabolic and RBF differences is not impaired by dense (fibro glandular) tissue, as this tissue is truly 
normal breast tissue and is what is present in $50 \%$ of all women FMTVDM measurement of fibro glandular tissue is measured as normal tissue as it is truly normal - absent some actual inflammation or cancer. As such, this dense tissue measures as normal fibro glandular (dense) breast tissue.

Similarly, FMTVDM is not impaired by breast implants as the implants are not supplied by the regional blood flow (RBF) of the breast, nor are the implants metabolically active. Any leakage of material from the implant into the breast tissue may be associated with increased RBF and metabolic activity within the breast - both not within the implant proper.

However, abnormal tissue, including cancerous and precancerous tissue as was present here significantly increased metabolic activity and regional blood flow (Figure 1); increased RBF and metabolism which can be measured independent of the presence of breast implants and increased breast density [2-4]. In this instance, the measured FMTVDM value confirmed that the abnormal tissue irregularity was in fact precancerous tissue.

\section{CONCLUSION}

While both dense breasts and breast implants produce problems for mammographic evaluation of breast tissue, including irregularities of the breast as was present in this case they do not affect RBF and metabolic differences measured by FMTVDM, which was able to identify the breast irregularities as precancerous changes in breast tissue (Figure 1).

\section{ACKNOWLEDGMENT}

FMTVDM is a utility patent issued to first author. All figures reproduced with expressed consent of first author. The authors wish to thank David L Prothro, MD of Nevada Arrhythmia Services Inc. in Reno, Nevada for independently conducting patient studies and providing results. The authors also wish to thank all the women and men with dense breasts and to Joe and Nancy Cappello, PhD.

\section{REFERENCES}

1. Bizjak M, Selmi C, Praprotnik S, Bruck O, Perricone C, et al. (2015) Silicone implants and lymphoma: The role of inflammation. J Autoimmun 65: 6473.

2. De Boer M, Colaris M, van der Hulst RRWJ, Cohen Tervaert JW (2017) Is explantation of silicone breast implants useful in patients with complaints? Immunol Res 65(1): 25-36.

3. Orciani M, Sorgentoni G, Olivieri F, Mattioli BM, Benedetto GD, Primio RD (2017) Inflammation by breast implants and adenocarcinoma: Not always a bad company. Clin Breast Cancer 17(4): 286-292.

4. Cohen TJW, Colaris MJ, van der Hulst RR (2017) Silicone implants and autoimmune rheumatic diseases: myth or reality. Curr Opin Rheumatol 29(4): 348-354.

5. Leberfinger AN, Behar BJ, Williams NC (2017) Breast implant-associated anaplastic large cell lymphoma: A systematic review. JAMA Surg 152(12): 1161-1168.

6. Collett DJ, Rakhorst H, Lennox P, Magnusson M, Cooter R, et al. (2019) Current risk estimate of breast implant-associated anaplastic large cell lymphoma in textured breast implants. Plast Reconstr Surg 143(S3): 30S-40S.

7. The Fleming Method for Tissue and Vascular Differentiation and Metabolism (FMTVDM) using same state single or sequential quantification comparisons. Patent Number 9566037.

8. Fleming RM, Dooley WC (2002) Breast enhanced scintigraphy testing distinguishes between normal, inflammatory breast changes and breast cancer: a prospective analysis and comparison with mammography. Integr Cancer Ther 1(3): 238-245.

9. Fleming RM, Fleming MR (2019) The importance of thinking about and quantifying disease like cancer and heart disease on a "Health-Spectrum" continuum. J Compr Cancer Rep 3(1): 1-3. 